COPD patients in the study at 6-months (day 182). CID was defined as: decrease of $\geq 100 \mathrm{~mL}$ in post-bronchodilator $\mathrm{FEV}_{1}$, or increase of $\geq 4$ units in the SGRQ, or a moderate/severe exacerbation. Using day 182 status, we tested the association between the occurrence of any CID type at or before 6 months and outcomes over the next 30 months including: sustained deterioration in $\mathrm{FEV}_{1}$ and SGRQ scores, moderate/severe exacerbations and mortality. A Cox's proportional hazards model used day 182 deterioration status with covariates collected at day 182, smoking status and geographical region to estimate future risk.

Results By day 182, 2870 [54\%] patients had experienced a CID $(\mathrm{CID}+)$ and 2422 [46\%] had not (CID-). 30 months later, the CID- group had a LS mean post-bronchodilator $\mathrm{FEV}_{1} 117 \mathrm{ml}$ (95\% CL 100,134; $\mathrm{p}<0.001$ ) higher compared to the CID+ group and the SGRQ total score was 6.4 units (95\% CL 5.4, $7.5 ; \mathrm{p}<0.001)$ better. Over the same period, post CID+ patients had a 61\% (95\% CL 50, 72\%; p < 0.001) increased risk of a new moderate/severe exacerbation and a $41 \%$ (95\% CL $15,72 \% ; \mathrm{p}<0.001)$ increased risk of all-cause death vs. the CID- group (see Figure 1).

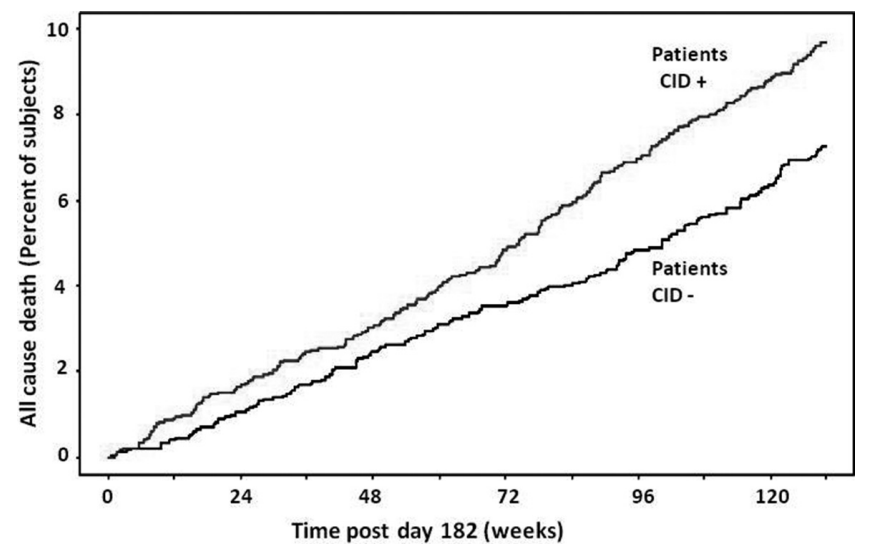

Abstract S57 Figure 1

Conclusion Patients experiencing a clinically important deterioration early in the TORCH trial appeared to be set on a clinical path of sustained deterioration in both health status and $\mathrm{FEV}_{1}$ with an increased medium/long-term future risk of exacerbations and all-cause death.

\section{S58 THE PEARL SCORE PREDICTS 90 DAY READMISSION OR DEATH FOLLOWING HOSPITALISATION FOR AN EXACERBATION OF COPD}

${ }^{1} \mathrm{C}$ Echevarria, 'I Steer, ${ }^{2} \mathrm{~K}$ Heslop-Marshall, ${ }^{2} \mathrm{SC}$ Stenton, ${ }^{3} \mathrm{PM}$ Hickey, ${ }^{3} \mathrm{R}$ Hughes, ${ }^{4} \mathrm{M}$ Wijesinghe, ${ }^{5} \mathrm{RN}$ Harrison, ${ }^{6} \mathrm{~N}$ Steen, ${ }^{6} \mathrm{AJ}$ Simpson, ${ }^{6} \mathrm{GJ}$ Gibson, ${ }^{1} \mathrm{SC}$ Bourke. ${ }^{1}$ North Tyneside General Hospital, Newcastle, UK; ${ }^{2} R V I$, Newcastle, UK; ${ }^{4}$ Royal Cornwall Hospital, Cornwall, UK; ${ }^{5}$ University Hospital of North Tees, Stockton-on-Tees, UK; ${ }^{6}$ Newcastle University, Newcastle, UK; ${ }^{3}$ Northern General Hospital, Sheffield, UK

\subsection{6/thoraxjnl-2015-207770.64}

Background Exacerbation of COPD is the second commonest reason for hospital admission, with high subsequent readmission and mortality rates among those who survive to discharge. Risk stratification would inform efficient use of services.

Methods Consecutive patients admitted with an exacerbation of COPD who survived to discharge were recruited by screening admissions units and searching coding records. Six UK hospitals took part: the derivation and internal validation cohort involved the same two hospitals at different time periods, and the external validation involved four hospitals.

Clinical data, and 90-day death and readmission rates were recorded. Multivariate logistic regression analysis was used to develop a tool to predict 90-day readmission, or death without readmission. Performance was assessed by the area under the receiver operator characteristic (AUROC) curve.

Results 2,417 patients were analysed (derivation 824, internal validation 824, external validation 791). Female 54.0\%, mean (SD) age 72.6 (10.2) years, FEV1 45.3 (18.2) \%predicted, 90day readmission or death $38.7 \%$.

In the derivation cohort, the five strongest predictors (odds ratio, 95\% confidence interval given for whole population) were: two or more Previous admissions in the preceding year (OR 3.17, 95\% CI 2.59-3.87), stable-state dyspnoea assessed by the Extended MRCD score (eMRCD 4 OR 1.46, 95\% CI 1.121.90; eMRCD 5a OR 2.35 95\% CI 1.79-3.08; eMRCD 5b OR $3.0095 \%$ CI 2.19-4.11), Age 80 or more (OR 1.48, 95\% CI 1.22-1.81), cor-pulmonale "Right heart failure" (OR 1.93, 95\% CI 1.41-2.66), Left heart failure (OR 1.45, 95\% CI 1.07-1.97). Two or more previous admissions and eMRCD $5 \mathrm{~b}$ were assigned a score of 3, eMRCD 5a scored 2, while eMRCD 4 and remaining indices scored 1. The risk of readmission and/or death is shown in Table 1.

The AUROC was: derivation 0.73 (95\% CI 0.69-0.77); internal validation 0.68 (95\% CI $0.64-0.72)$; and external validation 0.70 (95\% CI 0.66-0.73).

\begin{tabular}{|c|c|c|c|c|c|}
\hline Risk & Score & $\begin{array}{l}\text { Readmission or } \\
\text { death within } \\
90 \text { days }\end{array}$ & $\begin{array}{l}\text { Death within } \\
90 \text { days of } \\
\text { discharge }\end{array}$ & $\begin{array}{l}\text { Readmission } \\
\text { within } 90 \text { days of } \\
\text { discharge }\end{array}$ & Total \\
\hline \multirow[t]{4}{*}{ Low } & $0 \mathrm{n}$ & 54 & 7 & 50 & 343 \\
\hline & $\%$ & 15.7 & 2.0 & 14.6 & \\
\hline & $1 \mathrm{n}$ & 130 & 15 & 125 & 547 \\
\hline & $\%$ & 23.8 & 2.7 & 22.9 & \\
\hline \multirow[t]{6}{*}{ Intermediate } & $2 n$ & 164 & 46 & 149 & 462 \\
\hline & $\%$ & 35.5 & 10.0 & 32.3 & \\
\hline & $3 n$ & 155 & 58 & 125 & 352 \\
\hline & $\%$ & 44.0 & 16.5 & 35.5 & \\
\hline & $4 \mathrm{n}$ & 135 & 33 & 120 & 264 \\
\hline & $\%$ & 51.1 & 12.5 & 45.5 & \\
\hline \multirow[t]{12}{*}{ High risk } & $5 \mathrm{n}$ & 138 & 24 & 127 & 224 \\
\hline & $\%$ & 61.6 & 10.7 & 56.7 & \\
\hline & $6 \mathrm{n}$ & 90 & 28 & 83 & 131 \\
\hline & $\%$ & 68.7 & 21.4 & 63.4 & \\
\hline & $7 \mathrm{n}$ & 53 & 14 & 49 & 75 \\
\hline & $\%$ & 70.7 & 18.7 & 65.3 & \\
\hline & $8 \mathrm{n}$ & 11 & 6 & 10 & 13 \\
\hline & $\%$ & 84.6 & 46.2 & 76.9 & \\
\hline & $9 \mathrm{n}$ & 6 & 3 & 6 & 6 \\
\hline & $\%$ & 100 & 50 & 100 & \\
\hline & Total & 936 & 234 & 844 & 2417 \\
\hline & $\%$ & 38.7 & 9.7 & 34.9 & \\
\hline
\end{tabular}

Discussion In patients hospitalised with an exacerbation of COPD the PEARL score is a robust predictor of readmission and death and may be used to inform efficient use of resources according to risk. 